\title{
Front Matter: Volume 8307
}

, "Front Matter: Volume 8307," Proc. SPIE 8307, Passive Components and Fiber-Based Devices VIII, 830701 (28 December 2011); doi:

10.1117/12.921895

SPIE Event: SPIE/OSA/IEEE Asia Communications and Photonics, 2011, Shanghai, China 


\title{
PROCEEDINGS OF SPIE
}

\section{Passive Components and Fiber-Based Devices VIII}

\author{
Bishnu P. Pal \\ Editor \\ 14-16 November 2011 \\ Shanghai, China \\ Cosponsored by \\ SPIE \\ IEEE Photonics Society \\ Optical Society of America \\ Chinese Optical Society \\ China Institute of Communications \\ Local Organizing Committee \\ Shanghai University \\ Best Student Paper Sponsors \\ RSoft Design Group \\ Thorlabs, Inc. \\ Published by \\ SPIE \\ Optical Society of America \\ IEEE Photonics Society
}


The papers included in this volume were part of the technical conference cited on the cover and title page. Papers were selected and subject to review by the editors and conference program committee. Some conference presentations may not be available for publication. The papers published in these proceedings reflect the work and thoughts of the authors and are published herein as submitted. The publisher is not responsible for the validity of the information or for any outcomes resulting from reliance thereon.

Please use the following format to cite material from this book:

Author(s), "Title of Paper," in Passive Components and Fiber-Based Devices VIII, edited by Bishnu P. Pal, Proceedings of SPIE-OSA-IEEE Asia Communications and Photonics, Vol. 8307 (SPIE, Bellingham, WA, 2011) Article CID Number.

ISSN 0277-786X

ISBN 9780819489555

Published by

SPIE

P.O. Box 10, Bellingham, Washington 98227-0010 USA

Telephone +1 3606763290 (Pacific Time) · Fax +1 3606471445

SPIE.org

Optical Society of America

2010 Massachusetts Ave., N.W., Washington, D.C., 20036 USA

Telephone +1 2022238130 (Eastern Time) • Fax +1 2022231096

OSA.org

IEEE Photonics Society

445 Hoes Lane, Piscataway, New Jersey, 08855 USA

Telephone +1 7325628434 (Eastern Time) • Fax +1 7325628434

IEEE.org

Copyright ( 9 2011, Society of Photo-Optical Instrumentation Engineers, Optical Society of America, and IEEE Photonics Society.

Copying of material in this book for internal or personal use, or for the internal or personal use of specific clients, beyond the fair use provisions granted by the U.S. Copyright Law is authorized by SPIE subject to payment of copying fees. The Transactional Reporting Service base fee for this volume is $\$ 18.00$ per article (or portion thereof), which should be paid directly to the Copyright Clearance Center (CCC), 222 Rosewood Drive, Danvers, MA 01923. Payment may also be made electronically through CCC Online at copyright.com. Other copying for republication, resale, advertising or promotion, or any form of systematic or multiple reproduction of any material in this book is prohibited except with permission in writing from the publisher. The CCC fee code is $0277-786 \mathrm{X} / 11 / \$ 18.00$.

Printed in the United States of America.

Publication of record for individual papers is online in the SPIE Digital Library.

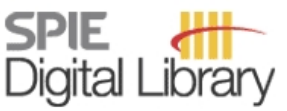

SPIEDigitalLibrary.org

Paper Numbering: Proceedings of SPIE follow an e-First publication model, with papers published first online and then in print and on CD-ROM. Papers are published as they are submitted and meet publication criteria. A unique, consistent, permanent citation identifier (CID) number is assigned to each article at the time of the first publication. Utilization of CIDs allows articles to be fully citable as soon as they are published online, and connects the same identifier to all online, print, and electronic versions of the publication. SPIE uses a six-digit CID article numbering system in which:

- The first four digits correspond to the SPIE volume number.

- The last two digits indicate publication order within the volume using a Base 36 numbering system

employing both numerals and letters. These two-number sets start with 00, 01, 02, 03, 04, 05, 06, 07, 08, 09, 0A, OB ... OZ, followed by 10-1Z, 20-2Z, etc.

The CID number appears on each page of the manuscript. The complete citation is used on the first page, and an abbreviated version on subsequent pages. Numbers in the index correspond to the last two digits of the six-digit CID number. 


\title{
Contents
}

\author{
xi Symposium Committee \\ xiii Conference Committee
}

SESSION 1 OPTICAL SIGNAL PROCESSING

830703 Time- and wavelength-interleaved laser pulses: prospects and challenges in optical signal processing (Invited Paper) [8307-02]

C. Shu, G. K. P. Lei, The Chinese Univ. of Hong Kong (Hong Kong, China)

$830704 \quad$ Novel multilayer structure CWDM demultiplexer in silica [8307-03]

A. Atieh, Taibah Univ. (Saudi Arabia); I. Mansour, Al-Ahliyya Amman Univ. (Jordan); Z. Dalala, Virginia Polytechnic Institute and State Univ. (United States)

830705 Enhanced slow light in a phase-shifted multichannel fiber Bragg grating assisted by stimulated Brillouin scattering [8307-04]

X. Chen, L. Xian, H. Li, Shizuoka Univ. (Japan)

830706 All-fiber high repetition rate short pulse generation around $1030 \mathbf{n m}$ [8307-05]

Q. Li, R. Zhu, C. Zhang, The Univ. of Hong Kong (Hong Kong, China); S. Yang, Tsinghua Univ.

(China); K. K. Y. Wong, The Univ. of Hong Kong (Hong Kong, China)

\section{SESSION 2 BEST STUDENT PAPER SESSION}

830707 A compact SOI polarization beam splitter based on multimode interference coupler [8307-06]

Z. Tu, Y. W. Huang, H. X. Yi, X. J. Wang, Y. P. Li, L. Li, W. W. Hu, Peking Univ. (China)

830708 Hybrid coupler with short range surface plasmon polariton and dielectric waveguide [8307-07]

B. Fan, F. Liu, R. Wan, Y. Huang, Tsinghua Univ. (China); Y. Miura, D. Ohnishi, ROHM Co., Ltd. (Japan)

830709 Gain property analysis of a quantum dot doped inner cladding fiber [8307-08]

Y. Dai, F. Pang, T. Wang, Shanghai Univ. (China)

8307 OA The graphene mode-locked fiber laser [8307-09]

S. Guo, A. Yang, Y. Sun, Beijing Institute of Technology (China)

8307 OB Ultra-compact and broadband orthogonal coupler between strip and slot silicon waveguides [8307-10]

F. Li, X. Hu, J. WU, L. Zhou, Y. Su, Shanghai Jiao Tong Univ. (China) 
8307 OC Coupling between dielectric waveguide mode and long range surface plasmon polariton waveguide mode (Best Student Paper Award) [8307-11]

Y. Li, F. Liu, B. Fan, R. Wan, Y. Huang, Tsinghua Univ. (China)

\section{SESSION $3 \quad$ ULTRAFAST OPTICS}

8307 OD Ultrafast laser inscription: science today, technology tomorrow (Invited Paper) [8307-12]

D. Choudhury, W. T. Ramsay, N. A. Willoughby, L. Paterson, A. K. Kar, Heriot-Watt Univ. (United Kingdom)

8307 OF Direct inscription of intrinsic Fabry-Perot interferometers in optical fiber tapers with a femtosecond laser [8307-14]

J. Li, X. Zhang, W. Wang, F. Pang, Y. Liu, T. Wang, Shanghai Univ. (China)

\section{SESSION $4 \quad$ FIBER LASERS AND AMPLIFIERS}

8307 OI Design and fabrication of an asymmetric twin-core fiber directional coupler for gain-flattened EDFA [8307-17]

B. Nagaraju, Indian Institute of Technology Delhi (India) and Sterlite Technologies Ltd. (India); M. Ude, S. Trzésien, B. Dussardier, Lab. de Physique de la Matière Condensée, CNRS, Univ. de Nice Sophia Antipolis (France); R. K. Varshney, Indian Institute of Technology Delhi (India); G. Monnom, W. Blanc, Lab. de Physique de la Matière Condensée, CNRS, Univ. de Nice Sophia Antipolis (France); B. P. Pal, Indian Institute of Technology Delhi (India) and Lab. de Physique de la Matière Condensée, CNRS, Univ. de Nice Sophia Antipolis (France)

$83070 \mathrm{~J} \quad$ Study of optical gain and noise characteristics in novel zirconia codoped erbium fibres [8307-18]

M. Pal, M. C. Paul, S. Das, R. Sen, S. K. Bhadra, Central Glass and Ceramic Research Institute (India)

8307 OK Investigation of single-polarized phosphate glass fiber laser and its self-pulsing behavior [8307-19]

F. Yang, Z. Pan, Q. Ye, H. Cai, R. Qu, Shanghai Institute of Optics and Fine Mechanics (China)

8307 OL Adiabatic soliton compression based on distributed Raman amplification in dispersion decreasing fiber [8307-20]

D. Jia, J. Chen, C. Wang, Y. Li, Z. Wang, T. Yang, Tianjin Univ. (China)

8307 OM A stable dual-wavelength single-longitudinal-mode fiber laser with a tunable wavelength spacing based on a chirped phase-shifted grating filter [8307-21]

M. Jiang, P. P. Shum, B. Lin, S. C. Tjin, Nanyang Technological Univ. (Singapore); Y. Jiang, Guizhou Univ. (China)

\section{SESSION $5 \quad$ SPECIALTY FIBERS, COMPONENTS, AND FIBER MEASUREMENTS I}

8307 ON Heavy metal fluoride glass fibers and their applications (Invited Paper) [8307-22]

M. Saad, IRphotonics Inc. (Canada) 
830700 Low power and compact eight-channel reconfigurable optical add-drop multiplexers based on cascaded microring resonators [8307-23]

Y. Tian, R. Ji, L. Zhang, J. Ding, H. Chen, L. Yang, Institute of Semiconductors (China)

8307 OP Compact water depth sensor with LPFG using the photoelastic effect and heat-shrinkable tube [8307-24]

S. Takama, T. Kudomi, M. Ohashi, Y. Miyoshi, Osaka Prefecture Univ. (Japan)

$8307 \mathrm{OQ} \quad \mathrm{Gb} / \mathrm{s}$ transmission of GI-HCS fiber with crimp and cleave connectors [8307-25]

X. Sun, J. Li, OFS (United States); B. Zhu, OFS Labs. (United States)

8307 OR Measurement of dispersion in a highly nonlinear fiber using four wave mixing [8307-26]

A. P. A., D. Venkitesh, Indian Institute of Technology Madras (India)

\section{SESSION 6 SPECIALTY FIBERS, COMPONENTS, AND FIBER MEASUREMENTS II}

8307 OS Parametric tunable dispersion compensator: distinctive features and practical issues (Invited Paper) [8307-27]

K. Tanizawa, J. Kurumida, T. Kurosu, S. Namiki, National Institute of Advanced Industrial Science and Technology (Japan)

8307 OT Radiation resistant optical fiber for visible region [8307-28]

A. Bhattacharya, A. Pal, Central Glass and Ceramic Research Institute (India);

G. K. Bhowmick, Bhabha Atomic Research Ctr. (India); A. Saha, UGC/DAE (India);

K. Dasgupta, R. Sen, Central Glass and Ceramic Research Institute (India)

8307 OU Sensitivity-enhanced optical temperature sensor with cascaded LPFGs [8307-29]

Y. Tsutsumi, Y. Miyoshi, M. Ohashi, Osaka Prefecture Univ. (Japan)

8307 OV Mid-infrared supercontinuum generation in arsenic trisulfide microstructured optical fibers [8307-30]

A. Jin, Z. Wang, J. Hou, B. Zhang, Z. Jiang, National Univ. of Defense Technology (China)

8307 OW Dopants concentration effects on the wavelength shift of long-period fiber gratings used as liquid level detectors [8307-32]

B.-M. Mao, B. Zhou, Zhejiang Univ. (China)

8307 OX Cladding-mode obtained by core-offset structure and applied in fiber Bragg grating sensor [8307-33]

X. Zhang, W. Peng, Y. Liu, H. Li, Z. Jing, Q. Yu, X. Zhou, W. Yao, Y. Wang, Y. Liang, Dalian Univ. of Technology (China)

\section{SESSION 7 MICRO-/NANO- FIBER/WIRE/WAVEGUIDE DEVICES}

8307 OY Optical microfiber passive devices and sensors (Invited Paper) [8307-34]

M. Ding, M. Belal, G. Chen, R. Al-Azawi, T. Lee, Y. Jung, P. Wang, Univ. of Southampton (United Kingdom); X. Zhang, Z. Song, National Univ. of Defense Technology (China); F. XU, Nanjing Univ. (China); R. Lorenzi, Univ. degli Studi di Milano-Bicocca (Italy); T. Newson, G. Brambilla, Univ. of Southampton (United Kingdom) 
830710 Coupling influence on the sensitivity of microfiber resonator sensors [8307-36]

W. Guo, Y. Chen, J. Kou, Nanjing Univ. (China); F. Xu, Nanjing Univ. (China) and Nanjing Univ. High-Tech Institute at Suzhou (China); Y. Lu, Nanjing Univ. (China)

830711 Temperature characteristics of microfiber coil resonators embedded in teflon [8307-37] Y. Chen, Y. Ming, W. Guo, Nanjing Univ. (China); F. XU, Nanjing Univ. (China) and Nanjing Univ. High-Tech Institute at Suzhou (China); Y. Lu, Nanjing Univ. (China)

830713 Design and fabrication of bilayer metallic nanowire polarizers and color filters based on surface plasmon and waveguide mode resonances [8307-39]

Z. Ye, J. Zheng, C. Zhang, S. Sun, Shanghai Jiao Tong Univ. (China)

830714 Transmission characteristics of a subwavelength metallic slit with perpendicular groove [8307-40]

L. Jin, J. Zhou, W. Zou, H. Zhang, L. Zhang, Ningbo Univ. (China)

\section{SESSION 8 NANOPHOTONICS}

$830716 \quad$ Nanophotonics inside structured optical fibres (Invited Paper) [8307-42]

J. Canning, The Univ. of Sydney (Australia)

830717 Theoretical analyses of localized surface plasmon resonance spectrum with nanoparticles imprinted polymers [8307-43]

H. Li, W. Peng, Y. Wang, L. Hu, Y. Liang, X. Zhang, W. Yao, Q. Yu, X. Zhou, Dalian Univ. of Technology (China)

\section{SESSION 9A METAMATERIALS, SILICON PHOTONICS, AND PLASMONICS}

8307 1A Optimizing the power confinement in silicon slot waveguides by use of finite element method [8307-46]

D. M. H. Leung, X. B. Kan, B. M. A. Rahman, N. Kejalakshmy, K. T. V. Grattan, City Univ. London (United Kingdom)

\section{SESSION 9B MICROWAVE PHOTONICS}

8307 1B Recent advances in millimeter-wave photonic wireless links for very high data rate communication (Invited Paper) [8307-47]

C.-L. Pan, National Tsing Hua Univ. (Taiwan, China); C. W. Chow, National Chiao Tung Univ. (Taiwan, China); C. H. Yeh, Industrial Technology Research Institute (Taiwan, China); C. B. Huang, National Tsing Hua Univ. (Taiwan, China); J. W. Shi, National Central Univ. (Taiwan, China)

8307 ID Cascaded microwave photonic filters with multiple infinite impulse responses based on wavelength conversion [8307-49]

E. XU, Nanjing Univ. of Posts and Telecommunications (China); F. Wang, Chongqing Univ. of Technology (China); L. Li, Nanjing Univ. of Posts and Telecommunications (China); Y. Yu, X. Zhang, D. Huang, Huazhong Univ. of Science and Technology (China) 
8307 IE Functional photonic crystal fiber sensing devices (Invited Paper) [8307-50]

J. Villatoro, V. Finazzi, Institute of Photonic Sciences (Spain); V. Pruneri, Institute of Photonic Sciences (Spain) and Catalan Institution for Research and Advanced Studies (Spain)

$8307 \mathrm{lH}$ Bandgap tunability of a liquid-filled photonic crystal fiber based on bend and temperature change [8307-53]

T.-T. Han, Y.-G. Liu, Z. Wang, Nankai Univ. (China); Y. Liu, Shanghai Univ. (China)

830711 Tapered photonic crystal fiber interferometer with enhanced sensitivity [8307-54]

S. Qiu, Y. Chen, J. Kou, Nanjing Univ. (China); F. Xu, Nanjing Univ. (China) and Nanjing Univ. High-Tech Institute at Suzhou (China); Y. Lu, Nanjing Univ. (China)

8307 1J A photonic crystal fiber for single-polarization single-mode operation [8307-55]

$H$. Zheng, Liaocheng Univ. (China) and Beijing Jiaotong Univ. (China); C. WU, Z. Wang,

Beijing Jiaotong Univ. (China); S. Liu, H. YU, X. Li, W. Wang, Z. Tian, Liaocheng Univ. (China)

\section{POSTER SESSION}

8307 1M Design of flat-top comb filter based on photonic crystal fiber Sagnac loop [8307-57]

X. Wang, S. Li, Huaibei Normal Univ. (China)

8307 iN Pressure/temperature sensor based on a dual-core photonic crystal fiber [8307-59]

D. Chen, G. Hu, Zhejiang Normal Univ. (China) and Joint Research Lab. of Zhejiang Normal Univ. and Zhejiang Univ. (China); L. Chen, Zhejiang Normal Univ. (China)

830710 Structure design of interleaver based on birefringent crystals [8307-60]

X. Pan, F. Luo, L. Deng, Huazhong Univ. of Science and Technology (China)

8307 IP Quantum-behaved particle swarm optimization for the synthesis of fibre Bragg gratings filter [8307-61]

X. Yu, Harbin Institute of Technology (China) and Harbin Univ. of Science and Technology

(China); Y. Sun, Y. Yao, J. Tian, S. Cong, Harbin Institute of Technology (China)

$83071 Q \quad$ Numerical analysis of the nonlinear polarization rotation mode-locked pulses in fiber [8307-62]

Z. Yang, Z. Yu, X. Zhang, Y. Song, Beijing Univ. of Technology (China)

8307 IR Coupling characteristics of a fluid-filled dual-core photonic crystal fiber based on temperature tuning [8307-63]

C. Wei, Z. Wang, Y. Liu, B. Liu, H. Zhang, Nankai Univ. (China); Y. Liu, Shanghai Univ. (China)

8307 is Bright-dark soliton pairs emission of a fiber laser [8307-64]

Y. Meng, S. Zhang, X. Li, H. Li, J. Du, Y. Hao, Hebei Normal Univ. (China)

8307 IT Stable CW operation in a ring fiber laser based on Er-doped photonic crystal fiber [8307-65]

C. Ouyang, P. P. Shum, K. Wu, Nanyang Technological Univ. (Singapore); M. Hu, L. Chai,

C. Wang, Tianjin Univ. (China); N. Dai, J. Li, Huazhong Univ. of Science and Technology

(China) 
8307 IV The tunable DBR fiber laser based on closed-loop PZT [8307-67]

M. Liu, Handan College (China) and Hebei Univ. Of Technology (China); C. Li, Hebei Univ. of Technology (China); G. Li, Y. Li, K. Yang, Handan College (China)

8307 IW Temperature and refractive index measurements using long-period fiber gratings fabricated by femtosecond laser [8307-68]

Y. Yu, J. Zheng, K. Yi, S. Ruan, C. Du, J. Huang, W. Zhong, Shenzhen Univ. (China)

$83071 \mathrm{X}$ Investigation of stimulated Brillouin scattering spectra of an optical fiber with use of a frequency modulated laser [8307-69]

M. A. Shaik, V. V. Achuth, D. Venkitesh, B. Srinivasan, Indian Institute of Technology Madras (India)

8307 IY A compact single-mode fiber ring depolarizer [8307-70]

W. Hao, C. Wang, L. Li, J. Wang, W. Fan, Shanghai Univ. (China)

830712 High energy passively mode-locked erbium-doped fiber laser at tens of kHz repetition rate [8307-71]

J. Chen, D. Jia, C. Wang, J. Wang, Z. Wang, T. Yang, Tianjin Univ. (China) and Key Lab. of Optoelectronics Information and Technical Science (China)

830720 Model of Bragg grating written in subwavelength-diameter fiber taper [8307-72]

P. Zhao, J. Zhang, Z. Wu, X. Zhang, Huazhong Univ. of Science and Technology (China)

830721 Nonlinear characterization of silver nanocrystals incorporated tellurite glasses for fiber development [8307-73]

Z. Zhou, Xi'an Institute of Optics and Precision Mechanics (China) and Graduate School of Chinese Academy of Sciences (China); W. Tan, J. Si, Xi'an Jiaotong Univ. (China); H. Zhan, J. He, Xi'an Institute of Optics and Precision Mechanics (China) and Graduate School of Chinese Academy of Sciences (China); A. Lin, Xi'an Institute of Optics and Precision Mechanics (China)

830722 Terahertz flat-top polarization filter based on liquid crystal cavities [8307-74] Y. Zhou, J. Zhang, P. GU, Shanghai Univ. (China)

830723 Design and analysis of multi-channel narrow-band polarization filter [8307-75]

P. GU, J. Zhang, Y. Zhou, Shanghai Univ. (China)

830724 Broadband optical antenna with a disk structure [8307-76]

I. Wang, Y. Du, The Hong Kong Polytechnic Univ. (Hong Kong, China)

830725 Calculation of ytterbium-doped double-cladding CW fiber amplifiers [8307-77]

X. Zhang, Y. Song, Z. Yu, X. Zhang, Beijing Univ. of Technology (China)

830726 Passively harmonic mode-locked fiber laser with controllable repetition rate based on a carbon nanotube saturable absorber [8307-78]

H. F. Li, S. M. Zhang, J. Du, Y. C. Meng, Y. P. Hao, X. L. Li, Hebei Normal Univ. (China)

830727 A compact and novel polarization beam splitter based on negative refractive photonic crystal slab [8307-79]

Z. XU, Y. Li, Peking Univ. (China) 
830729 Design and simulation of temperature-insensitive arrayed waveguide gratings based on silicon nanowires [8307-81]

Y. Chen, Zhejiang Univ. (China); T. Lang, Zhejiang Univ. (China) and China Jiliang Univ. (China); J. Zou, J.-J. He, Zhejiang Univ. (China)

8307 2A Fiber Bragg grating Fabry-Perot cavity sensor based on pulse laser demodulation technique [8307-83]

F. Gao, J. Chen, Y. Liu, T. Wang, Shanghai Univ. (China)

$83072 \mathrm{~B}$ Response of $\mathrm{CO}_{2}$ laser written long period fiber gratings packaged by polymer materials [8307-84]

Z. Wu, Y. Liu, J. Zou, N. Chen, F. Pang, T. Wang, Shanghai Univ. (China)

8307 2C Fabry-Perot cavity based on non-matching fiber Bragg gratings studied by V-I transmission matrix method [8307-85]

F. Li, Y. Liu, F. Gao, T. Wang, Shanghai Univ. (China)

8307 2D Fabrication of tilted long-period fiber gratings by $\mathrm{CO}_{2}$ laser [8307-86]

R. Wu, Y. Liu, J. Zou, N. Chen, F. Pang, T. Wang, Shanghai Univ. (China)

8307 2F Cascaded quadratic soliton compression by seeded second harmonic generation [8307-88]

X. Zhan, J. Hu, X. Zeng, T. Wang, Shanghai Univ. (China)

8307 2G A novel dispersion compensating fiber with multiple windows based on hybrid photonic crystal fiber [8307-90]

Y. Liu, Y. Li, R. Wang, J. Wang, Y. Su, X. Xie, The PLA Univ. of Science and Technology (China)

$83072 \mathrm{H} \quad$ Defect center characteristics of silica optical fiber material by gamma ray radiation [8307-91]

W. Luo, Shanghai Univ. (China) and Shanghai Applied Radiation Institute (China); Z. Xiao, J. Wen, J. Yin, Z. Chen, Z. Wang, T. Wang, Shanghai Applied Radiation Institute (China)

83072 Attenuated total reflectance (ATR) $\mathrm{GeO}_{2}$ hollow infrared waveguides deposited from aqueous germanate ion solutions with different $\mathrm{GeO}_{2}$ concentrations [8307-92]

Y. Li, Qingdao Univ. of Science and Technology (China); C. Jing, East China Normal Univ. (China); J. Chu, Shanghai Institute of Technical Physics (China)

$83072 \mathrm{~J}$ Influence of photo- and thermal bleaching on pre-irradiation low water peak single mode fibers [8307-93]

J. Yin, J. Wen, Shanghai Univ. (China); W. Luo, Shanghai Univ. (China) and Shanghai Applied Radiation Institute (China); Z. Xiao, Z. Chen, T. Wang, Shanghai Univ. (China)

8307 2K Optical ultra-wideband pulse generation based on incoherent optical arbitrary waveform generation [8307-94]

G. Zhang, X. Zheng, H. Wen, H. Zhang, B. Zhou, Tsinghua Univ. (China)

8307 2L Gas furnace design for low-temperature and low-speed fiber drawing process [8307-95]

Q. Guo, T. Wang, N. Chen, Z. Chen, Q. Zhou, M. Tang, Shanghai Univ. (China) 
8307 2M Design and fabrication of a 1-by-4 multimode interference splitter based on InP [8307-135] M. Li, Institution of Semiconductors (China) and Tsinghua Univ. (China); C. Zhang, H. Zhu, Institution of Semiconductors (China); M. Chen, Tsinghua Univ. (China)

$83072 \mathrm{~N} \quad$ Supercontinuum generation in polarization maintaining photonic crystal fibers using nanosecond pulses [8307-137]

Y. Yu, Y. Zhang, B. Zhang, Z. Wang, National Univ. of Defense Technology (China)

830720 Dispersion characteristics analysis of asymmetric multi-core fibers [8307-138] J. Gao, X. Zhang, L. Shi, W. Shi, Y. Huang, X. Ren, Beijing Univ. of Posts and Telecommunications (China)

8307 2P Design of a dual-parallel-core fiber for dispersion compensation [8307-139] W. Shi, X. Zhang, L. Shi, J. Gao, X. M. Ren, Y. Huang, Beijing Univ. of Posts and Telecommunications (China)

Author Index 


\title{
Symposium Committees
}

\author{
Honorary General Chairs
}

Tingye Li, AT\&T Laboratories (retired) (United States)

Bingkun Zhou, Tsinghua University (China)

General Chairs

Jian-Jun He, Zhejiang University (China)

Ken-ichi Kitayama, Osaka University (Japan)

Xingde Li, Johns Hopkins University (United States)

Technical Program Chairs

Perry Shum, Nanyang Technological University (Singapore)

Yikai Su, Shanghai Jiao Tong University (China)

Arthur Chiou, National Yang-Ming University (Taiwan, China)

Local Organizing Committee

Yunqi Liu, Shanghai University (China)

Ronghui Qu, Shanghai Institute of Optics and Fine Mechanics (China)

Tingyun Wang, Shanghai University (China)

Xiaobei Zhang, Shanghai University (China)

Steering Committee Chairs

Ming-Jun Li, Corning USA (United States)

John Zyskind, Oclaro, Inc. (United States)

Steering Committee Members

Sailing He, Zheijang University (China)

Peter Kaiser, Telcordia Technologies (retired)

Connie Chang-Hasnain, University of California, Berkeley (United States)

Chongcheng Fan, Tsinghua University (China)

Mao Qian, WRI-Fiberhome (China)

Xiaomin Ren, Bejing University of Posts \& Telecommunications (China) 
Downloaded From: https://www.spiedigitallibrary.org/conference-proceedings-of-spie on 26 Apr 2023

Terms of Use: https://www.spiedigitallibrary.org/terms-of-use 


\title{
Conference Committee
}

\author{
Conference Chair
}

Bishnu P. Pal, Indian Institute of Technology Delhi (India)

Conference Cochairs

Mable P. Fok, Princeton University (United States)

Aoxiang Lin, Xi'an Institute of Optics and Precision Mechanics (China)

Program Committee

Govind P. Agrawal, University of Rochester (United States)

Adrian L. G. Carter, Nufern (United States)

Lawrence R. Chen, McGill University (Canada)

Kin-Seng Chiang, City University of Hong Kong (Hong Kong, China)

Wei Jin, The Hong Kong Polytechnic University (Hong Kong, China)

Raman Kashyap, Ecole Polytechnique de Montréal (Canada)

Karl W. Koch, Corning Inc. (United States)

Byoungho Lee, Seoul National University (Korea, Republic of)

Xueming Liu, Xi'an Institute of Optics and Precision Mechanics (China)

Alan Rolf Mickelson, University of Colorado at Boulder (United States)

Gérard Monnom, Université de Nice Sophia Antipolis (France)

Suresh Nair, Network Systems \& Technologies (P) Ltd. (India)

Andrew W. Poon, Hong Kong University of Science and Technology (Hong Kong, China)

B. M. Azizur Rahman, The City University (United Kingdom)

Siddharth Ramachandran, The Boston University Photonics Center (United States)

Yun-Jiang Rao, University of Electronic Science and Technology of China (China)

Jayanta K. Sahu, University of Southampton (United Kingdom)

Ravindra Kumar Sinha, Delhi Technological University (India)

Fei Xu, Nanjing University (China)

Session Chairs

1 Optical Signal Processing

Bishnu P. Pal, Indian Institute of Technology Delhi (India)

2 Best Student Paper Session

Aoxiang Lin, Xi'an Institute of Optics and Precision Mechanics (China)

Bishnu P. Pal, Indian Institute of Technology Delhi (India) 
$3 \quad$ Ultrafast Optics

Fei Xu, Nanjing University (China)

$4 \quad$ Fiber Lasers and Amplifiers

B. M. Azizur Rahman, The City University (United Kingdom)

5 Specialty Fibers, Components, and Fiber Measurements I

Aoxiang Lin, Xi'an Institute of Optics and Precision Mechanics (China)

6 Specialty Fibers, Components, and Fiber Measurements II

Ajoy Kar, Heriot-Watt University (United Kingdom)

$7 \quad$ Micro-/Nano- Fiber/Wire/Waveguide Devices

B. M. Azizur Rahman, The City University (United Kingdom)

$8 \quad$ Nanophotonics

Fei Xu, Nanjing University (China)

9A Metamaterials, Silicon Photonics, and Plasmonics

Bishnu P. Pal, Indian Institute of Technology Delhi (India)

Ravendra K. Varshney, Indian Institute of Technology Delhi (India)

9B Microwave Photonics

Yange Liu, Nankai University (China)

10 Photonic Crystal Fiber and Devices

David N. Payne, University of Southampton (United Kingdom)

Joel Villatoro, Institut de Ciències Fotòniques (Spain)

Telecom Post-Deadline Papers: Joint Session with Conferences 8308, 8309 , and 8310

Naoya Wada, National Institute of Information and Communications Technology (Japan) 